\title{
OBSERVATIONS ON SARCOPTIC MANGE IN THE HORSE.
}

\author{
By Vet.-Lieut. R. Butler, A.V.D., Aldershot.
}

THE following details of the equine variety of sarcoptes scabiei are drawn from observation of some cases which have recently come under the notice of the writer, and it is hoped that the description of the parasite here given may be warranted by the apparent paucity of English literature on the subject.

History.- The history of the cases under notice is interesting. In the early spring of I 89 I two horses were admitted to hospital for eczema, and when seen by the writer some three months subsequently they were to all appearance perfectly healthy. One of these horses has been in constant work ever since, and at present is in particularly good condition. The other continued to work for six months, always, however, having a dry irritable condition of the skin, though never displaying any decided eruption. At the end of that period the case was again admitted for treatment of a slight eczematous-looking eruption, which easily yielded to appropriate remedies. After the lapse of another month attention was drawn to the profuse desquamation which was noticed from this animal, and shortly afterwards the horses on each side were found to have an eczematous eruption on the shoulders. Several microscopical examinations had previously been made without result, but on this occasion the acarus was detected, and subsequently a great number were observed.

The quiestion as to whether the disease had existed undetected for the whole of the period mentioned, or whether it had been subsequently contracted by an animal with a condition of skin rendering it peculiarly liable to contagion, is hard to decide; but having regard to the length of time which the parasites must have been in operation to produce the excessive epidermal desquamation, which was general over the whole body, it is probable that it did exist undetected, and that the well-known trouble in unearthing this particular variety of parasite, and the good hygienic conditions under which the animals were kept, combined to make its detection difficult and its effects comparatively slight.

Frequency of the Disease.-It would appear that this disease is not so infrequent in this country as has been generally supposed, for careful observation by others (Vet.-Capt. Smith and Vet.-Capt. Rutherford) has discovered two other cases, totally unconnected with the outbreak under notice or with each other, within the past two months; and in each the acarus has been discovered and identified. It is certain from the history of one of these cases that the disease may be present in a chronic form, causing comparatively little disturbance for many weeks or even months, and it is only by careful observation that its nature can be detected. It is stated by Robcrtson (Equine MTed.) that sarcoptic scabies is rather less frequent than the psoroptic variety, but no data are advanced in support of the statement.

Macroscopical Appearances.-The starting point of the disease in all cases observed is the shoulder or withers, from whence it spreads along the back, up the neck, down the quarter and chest, and ultimately on to the limbs. 
On attention being drawn to the patient, it is found that the part affected is covered by a discrete cruption, consisting of small nodules of dried serum, easily detachable, and each embracing several hairs, which come away with the crust, leaving a circular slightly moist patch. This is the eczema of sarcoptic scabies, easily distinguishable from the diffuse, more humid and circumscribed areas produced by the psoroptic variety, but which is most likely, as pointed out by various authors, to be mistaken for non-parasitic eczema.

A small blood spot may occasionally be noted in the centre of the bare surface, but its presence is by no means constant, nor indeed common.

Pruritus, though not in a very marlied degree, is a constant accompaniment; and, as the disease progresses, wrinkling of the skin and clesquamation of the epidermis are noticeable features.

Pruritus, in the cases under notice, was not the severe symptom described by the authors to whom reference is made below.

Neumann (Maladies Parasitaires) observes "The first sign of the disease is itching; the animal chafes itself against surrounding objects; gnaws at such parts as are within reach; enjoys the friction of the curry-comb during grooming; and by leaning towards the groom, tries to increase its action. If scratched, it betrays its satisfaction by characteristic movements of the nostril," etc.

Edgar (Procedings of Sezenth Gencral llecting of $N^{\top} . T^{\top}$.A.) states, "Scabies is distinguished from the various forms of eczema and psoriasis by the intense itching;" whilst Fleming (San. Sci. and Police) observes, "The first visible symptom is that occasioned by itching, which is so intense that the horse is incessantly gnawing at the affected parts, scratching them with its hoofs and rubbing them against the wall," etc. In all the cases under observation itching undoubtedly existed, and by its presence first attracted attention, but it was not marked by the severity above described, nor were any of the patients chafed from rubbing themselves. When the skin was scratched there was slight champing of the jaws and eversion of the upper lip, and in one case crouching of the loins, but these were the only signs evoked. The description given by Fleming, quoted above, seems more applicable to the psoroptic form, where the extreme irritation and the savage pleasure derived from rubbing the part affected are peculiarly marked; but the same author states that "it is not a matter for doubt that horses when stabled, and well or even moderately fed, will resist the irritation without exhibiting much disturbance in health, but it is quite otherwise when they are exposed to hardships ; "and this may perhaps account for the apparent discrepancy in observation, as the cases under notice were, and always had been, under good hyogienic conditions.

Wrinkling of the skin is a persistent concomitant of the disease in the more advanced stages, when it is found first upon the neck, subsequently extending over the ribs and shoulders, and even being occasionally marked at the point of the elbow and over the stifle.

This curious vertical folding of the skin is noticed by all authors, and some stress has been laid upon it as a diagnostic symptom of the disease. As this condition is observed in another skin affection, viz. that which is so common in India and which is now believed to be due to a vegetable parasite ; and as, moreover, it is induced or at any 
rate severely aggravated by the application of irritating dressings, notably the oil of tar, it cannot be regarded as absolutely diagnostic, but in conjunction with others is entitled to serious consideration.

The subsequent effects of the parasitic irritation of the hair follicles are most marked; small patches of darker coloured hair than normal, and which grow very rapidly, are studded all over the parts which were affected, and the appearance of a recovered case shows us that no part must be suffered to escape treatment, for small islands of these dark hairs will be found dotted about in places remote from the chief seat of disease, and totally unsuspected previous to treatment.

Microscopical Apperronces.-The parasite and its eggs are found imbedded in the epidermal scales, from which they must be separated by the addition of reagents before casy observation is feasible, a drop of Liq. Potassæ. being sufficient to effect this.

The eggs are ovoid and slightly granular; as development progresses this granular condition becomes concentrated at either end, and, later, at these points of concentration the divisions of the head and legs may

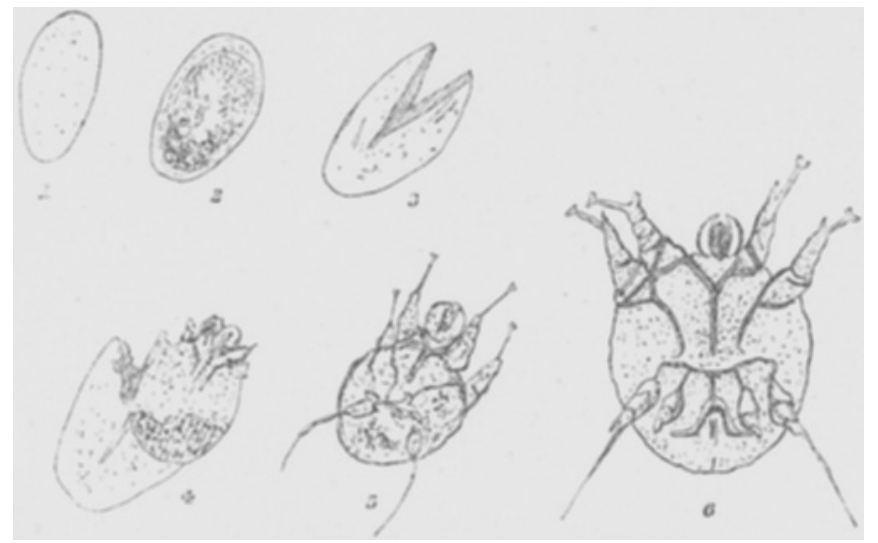

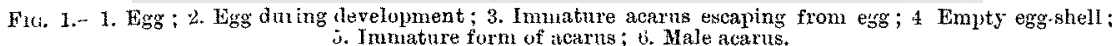

be made out, the latter being telescoped inwards upon the body. The egg is then spht up vertically for about half its length to admit of the escape of the young acarus.

The immature acarus is distinguished from the mature by the possession of only one pair of hind legs. The change to maturity is accompanied by a cast of skin, from which the insect emerges with its full complement of limbs. This change, which $I$ have not been fortunate enough to observe, is well figured in Neumann's Maladies Parasitaires.

In the mature female the body is egg-shaped, and the dorsal surface is covered with backwardly projecting spines, which are arranged as follows: (I.) Centrally situated are many small, triangular ones, which are also seen here and there over the greater part of the back. (2.) Anteriorly situated to these, on either side are a few long, thorn-like hairs ; (3.) and posteriorly, are four rows of similar long hairs, each row consisting of thrce, vertically arranged. The fore legs, two on each side of the head, are short pyramidal and telescopically jointed, the joints being three in number. From the inner angle of the base of each limb, a long, sharply defined process extends along the ventral 
PLATE I

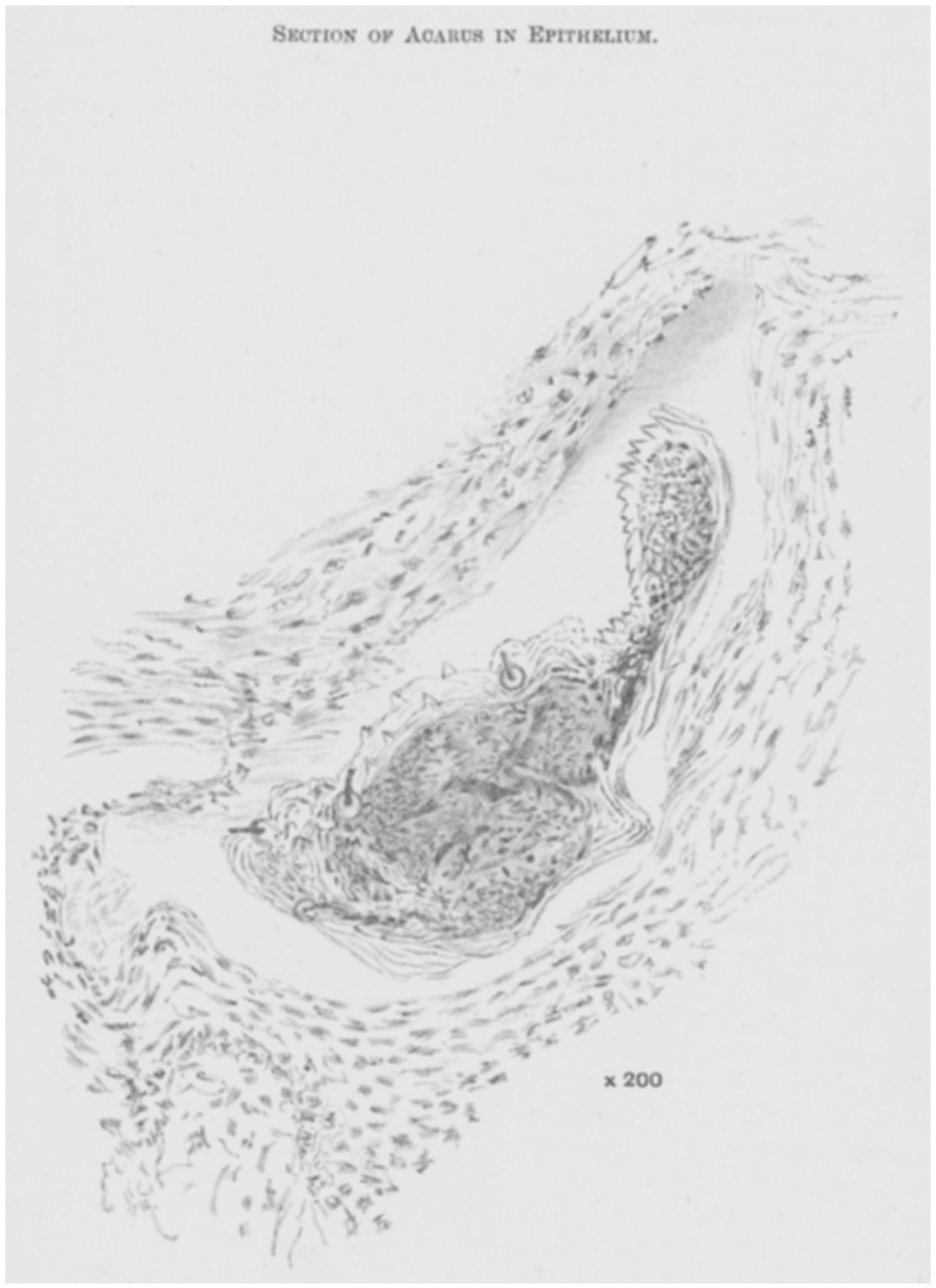


surface towards the centre of the insect, the two central processes jointing and being continued together. At the apex of each limb is
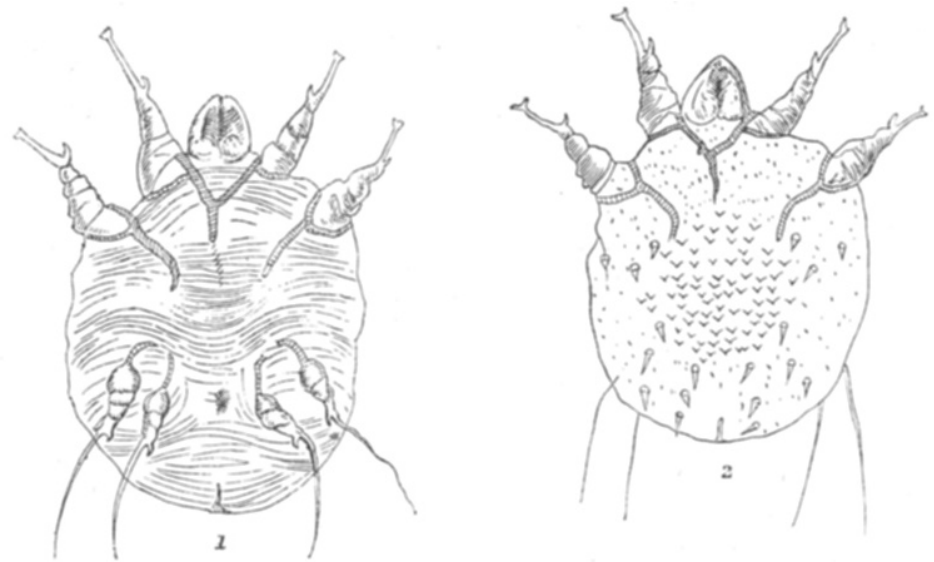

Fia. 2.-1. Female acarus, ventral surface ; 2. Female acarus, dorsal surface, showing the pectiar arrangement of the spines. The objects in this and the preceding figure are represented as seen under Zeiss's BB objective and No, 4 ocular.

a strong, sharp hook, and a bell-shaped sucker is attached to a hairlike prolongation of the terminal joint.

The hind legs consist of two pairs, placed rather widely apart and wholly underneath the body, so that when the parasite is on its ventral surface they are totally concealed. . Like the fore limbs, each has a strong terminal hook, but in place of a sucker a long curved hair is found, and from the base of each limb a sharply defined process extends towards the centre of the acarus.

The head is short, almost spherical, and devoid of a neck; the mandibles are sharp, strong, and curved towards each other.

The male is from $\frac{1}{2}$ to $\frac{2}{3}$ the size of the female; the body is more circular, but few spines are observed on the back, and the outer pair of hind limbs project slightly from the body. Between the inner hind limbs is seen the large $\mathbf{V}$-shaped organ of copulation.

The drawings which accompany these observations are made from various specimens in my possession, are magnified about Ioo times, and illustrate the development of the parasite according to the description already given. The detection of the acarus is attended with some difficulty owing to the depth at which it is found in the skin. They may, however, be readily observed in the scales from cases of any duration; and even, when the parasite itself is not detected, the presence of eggs and excreta is sufficient to warrant a certain diagnosis.

\title{
SARGOPTIC SCABIES IN THE HORSEx
}

\author{
By Vet.-Capt. C. Rutherford, A.V.D., Aldershot.
}

PORTIONS of skin removed by Vet.-Lieut. Butler from troop horses, Nos. B 52 and C I 3, 5 th Dragoon Guards, known to be suffering from the sarcoptic form of scabies, were kindly submitted to me for microscopical examination. Immediately after removal from the horses, these were placed in spirit, and subsequently embedded in celloidin. 